\title{
So Now What? Unanswered Questions Regarding Retroperitoneal Sarcomas, Hospital Volume, Multidisciplinary Expertise, and Outcomes
}

\author{
Alessandro Gronchi, $\mathrm{MD}^{1}$ (D), and Chandrajit P. Raut, $\mathrm{MD}^{2}$ \\ ${ }^{1}$ Department of Sugery, Fondazione IRCCS Istituto Nazionale dei Tumori, Milan, Italy; ${ }^{2}$ Department of Surgery, Brigham \\ and Women's Hospital, Center for Sarcoma and Bone Oncology, Dana-Farber Cancer Institute, Harvard Medical School, \\ Boston, MA
}

We commend Dr. Villano and colleagues for undertaking this important appraisal of modern variabilities in therapeutic strategies between low- and high-volume retroperitoneal sarcoma (RPS) centers. ${ }^{1}$ The authors used the National Cancer Database (NCDB) to demonstrate a trend toward increased use of preoperative radiotherapy (RT) and multi-visceral resections in centers performing a low volume of primary RPS resections, bringing them closer to practicing patterns in place at high-volume centers. Whether the trend toward adoption of these practices translated into better outcomes was not investigated. Thus, whether the observed trend has led to a consistent improvement in the quality of care that RPS patients receive across the United States remains uncertain. One challenge is the difficulty of objectively identifying why patients treated at high-volume RPS centers have better results than those treated at low-volume RPS centers.

We assume that team characteristics, experience, and expertise in patient selection, local therapies, and multidisciplinary strategies play critical roles, as do institutional characteristics such as ability to rescue after a complication. Nonetheless, factors such as size of the country, insurance policies, and patient preference have resulted in

(C) Society of Surgical Oncology 2021

First Received: 15 October 2021

Accepted: 23 November 2021;

Published Online: 9 January 2022

A. Gronchi, MD

e-mail: alessandro.gronchi@istitutotumori.mi.it
RPS care remaining balkanized rather than regionalized although data supporting the need to regionalize RPS care are available and in fact compelling., 2,3

The high proportion of patients in the United States who do not have their primary RPS resected at a high-volume center is worrisome. Previous studies ${ }^{2,3}$ have demonstrated that higher case volume is associated with better survival. In the current study, only $9.3 \%$ of the patients undergoing surgery for primary RPS had their surgery at a high-volume hospital (HVH) between 2004 and 2017, similar to the $9.8 \%$ rate reported previously by Keung et al. ${ }^{3}$ in a NCDB study spanning an overlapping period (1998-2011). However, a threshold value for case volume has not been clearly identified to date.

In the current study, ${ }^{1}$ the definition of $\mathrm{HVH}$ was based on a threshold of more than 10 cases per year (the same as in the prior Keung et al. ${ }^{3}$ study). A previous study performed by the same authors had identified a target of 13 cases per year. ${ }^{4}$ However, these numbers should be viewed with caution because very few centers contributing to the NCDB manage more than 10 cases per year. In addition, the mean number of cases treated at HVHs was 30 per year in the prior study and 18 per year in this new study. The reported proportional improvement in overall survival (OS) by the increased number of cases per year, which plateaued after 13 cases per year in the first study, may well be related to centers that treat a significantly higher number of cases. Therefore, a real data-driven threshold remains uncertain.

In Europe, a consensus document proposed the performance of 30 to 40 cases per year as one of the requirements for an expert sarcoma surgeon and 100 new cases per year as the threshold for a sarcoma center, including nonsurgical 
cases. $^{5}$ A threshold of 25 cases of primary RPS cases per year also was proposed for abdominal sarcoma referral centers. However, to date, no data exist to show what properties should define a "sarcoma referral center." Furthermore, it is even less clear whether the experience of a sarcoma referral center with high-volume expertise in certain types of sarcoma (e.g., extremity sarcoma or gastrointestinal stromal tumor [GIST]) can automatically be translated to other types of sarcomas (RPS).

Consensus exists about the need to have multidisciplinary tumor boards dedicated to the disease. Consensus also exists about the need to identify a minimum case volume. However, a uniform threshold remains to be identified and undoubtedly will be influenced by the country's overall population and geography, as well as by cultural and health care-related issues. However, this begs the question the current study tried to answer: can we compensate for the lack of regionalized referral by fostering the adoption of the same therapeutic strategies at all centers? In other words, is it reassuring to see that lowvolume hospitals (LVHs) are trending toward the strategies of HVHs or is it even more worrisome? Institutional experience has demonstrable value that cannot be matched by the simple application of recommendations alone.

Derbel et al. ${ }^{6}$ conducted a study in the Rhone Alpe region of France, which showed how surgery adherent to clinical practice guidelines was statistically associated with a better outcome only if performed in an expert referral center. Whether this also applies to other local methods such as RT is uncertain.

A trend toward the adoption of more preoperative RT and multi-visceral resections in LVHs, which is the focus of this report, still may not improve outcomes without the additional presence of a specific expertise for the disease. This critical question is not addressed by this report. What clearly is needed at this point is an analysis exploring the possibility of improvement in outcomes over time at LVHs in concert with the increased adoption of more preoperative RT and multi-visceral resections. The median follow-up period of the current series may not have been sufficient to identify differences in OS, the only relevant end point that can be measured by the NCDB. Better local strategies have a direct impact on local control, which may translate into a survival benefit only at a later stage. Given this limitation, even if changes in survival outcome of LVHs and HVHs over time had been calculated, the long-term impact on survival would potentially have gone undetected. This, in turn, raises the question about the validity of using such a large national dataset to tackle this important question.

Indeed, the NCDB is useful for studying several common cancers. However, it has several limitations when it comes to the understanding of rarer tumors such as sarcomas given the complexity of the disease and the lack of key information in the database. A global effort to study RPS (the Transatlantic Australasian RetroPeritoneal Sarcoma Working Group) currently is underway, and as of January 2017, a prospective registry has been established. This registry is designed to address many specific questions about RPS care and will be critical for improving the knowledge in the coming years.

Moreover, it is difficult to assess quality of care simply by measuring the percentage of multi-visceral resections and/or use of adjuvant/neoadjuvant treatments. Multi-visceral resection in this report (and in the NCDB) is defined as removal of the tumor en bloc with at least part of one organ. Indeed, multi-visceral resection is a definition that can be interpreted in many different ways and as such is not indicative of an adequate operation.

Current consensus standards in RPS surgery ${ }^{7,8}$ have been proposed and published in recent years. Appropriate surgery is not simply about the en bloc resection of multiple organs with the tumor. It is indeed true that removal of a mass without resection of any surrounding organ/structure is rarely adequate in RPS.

In a large series of primary RPS treated at multiple international referral institutions, ${ }^{9}$ the percentage of resections without removal of any surrounding structure/ organ was only $10.5 \%$, strikingly lower than the rate of $55 \%$ (56\% in $\mathrm{LVH}$ and $46 \%$ in $\mathrm{HVH}$, respectively) in the current NCDB series during the same period. Therefore, many resections very likely were inadequate. However, surgical procedures should be tailored to histologic type together with anatomic presentation and direct invasion of the adjacent organs. ${ }^{10}$ For instance, retroperitoneal (RP) \liposarcoma (LPS) resection commonly requires at least removal of the ipsilateral kidney, usually together with the ipsilateral colon. Part of the ipsilateral psoas muscle, and more rarely of the diaphragm, also may be needed. In addition, for left-sided RP LPS, resection of the spleen and body/tail of the pancreas will be required in approximately $50 \%$ of the cases, whereas for right-sided LPS, resection of the duodenum/head of the pancreas is needed in only $2 \%$ to $3 \%$ of the cases. Finally, vascular resection or bone resection is necessary in $5 \%$ to $10 \%$ of the cases. Therefore, for RP LPS, the most common histologic type at this site, a simple excision of the mass is practically never adequate. Beyond that, resection of the tumor with part of one organ, as multi-visceral resection is defined in this report, likely is not adequate either.

For RP leiomyosarcoma (LMS), resection requires a less extended procedure, which depends on vascular involvement because retroperitoneal LMS often originates from major retroperitoneal veins (inferior vena cava, renal veins, gonadal veins, iliac veins). For solitary fibrous tumor, resection often can be performed instead without the need to resect surrounding organs. Retroperitoneal malignant 
peripheral nerve sheath tumor (MPNST) often, if not always, originates from the femoral nerve and lies between the spine and the psoas muscle. Resection of MPNST thus typically requires removal of the nerve of origin and the immediately adjacent psoas muscle without resection of any visceral organ. For other rarer histologic types, the approach will depend upon the presentation and direct organ involvement. Thus, simply using multi-visceral resection as a surrogate for adequate surgery is very inaccurate. These variegated approaches to histology-specific RPS resection require multiple surgical skills on the one hand and a deep expertise in disease biology on the other hand, together with ability to discuss the best approach within dedicated sarcoma-focused multidisciplinary tumor boards.

Finally, having LVH institutions adopt HVH strategies only in the surgical realm is not sufficient. Great expertise in delivering RT to minimize toxicity while adequately treating the operative bed's radial margins is required. Furthermore, the recent STRASS trial, the first randomized trial evaluating preoperative RT plus surgery versus surgery alone for primary RPS, demonstrated an overall lack of benefit from RT, with some signals suggesting that preoperative RT may be justifiably considered for welldifferentiated LPS. ${ }^{11}$ Thus, HVHs may now scale back their use of preoperative RT, just as LVHs are using RT more. If those LVHs lack sufficient expertise in delivery of RT from dedicated sarcoma radiation oncologists, this desynchrony could expose patients at LVHs not only to RT without therapeutic benefit (if the teams at the LVHs are not aware of the published data), but also to toxicity from imprecisely delivered RT.

We believe that although understanding how patients are treated at a country level is important, every effort should be made to foster regionalization to high-volume RPS centers. The importance of experience in resection of other malignancies, such as pancreatic and esophageal cancers, has been recognized for decades. It is time to encourage better regionalization for RPS resections, acknowledging the various financial and travel challenges this poses. This will be the "simplest" and only way to improve quality of care and hence the chance of cure for RPS in the years to come.

DISCLOSURES There are no conflicts of interest.

\section{REFERENCES}

1. Villano AM, Vidri RJ, Vo ET, et al. National trends in treatment for retroperitoneal soft tissue sarcoma: a modern appraisal of variability in therapeutic strategies. Ann Surg Oncol. 2021. http s://doi.org/10.1245/s10434-021-10908-z.

2. Bonvalot S, Gaignard E, Stoeckle E, et al. Survival benefit of the surgical management of retroperitoneal sarcoma in a reference center: a nationwide study of the French Sarcoma Group from the NetSarc database. Ann Surg Oncol. 2019;26:2286-93.

3. Keung EZ, Chiang YJ, Cormier JN, et al. Treatment at lowvolume hospitals is associated with reduced short-term and longterm outcomes for patients with retroperitoneal sarcoma. Cancer. 2018;124:4495-503.

4. Villano AM, Zeymo A, Chan KS, et al. Identifying the minimum volume threshold for retroperitoneal soft tissue sarcoma resection: merging national data with consensus expert opinion. $J \mathrm{Am}$ Coll Surg. 2020;230:151-60.

5. Andritsch E, Beishon M, Bielack S, et al. ECCO essential requirements for quality cancer care: soft tissue sarcoma in adults and bone sarcoma: a critical review. Crit Rev Oncol Hematol. 2017;110:94-105.

6. Derbel O, Heudel PE, Cropet C, et al. Survival impact of centralization and clinical guidelines for soft tissue sarcoma (a prospective and exhaustive population-based cohort). PLoS One. 2017;12:e158406.

7. Management of primary retroperitoneal sarcoma (RPS) in the adult: a consensus approach from the Trans-Atlantic RPS Working Group. Ann Surg Oncol. 2015;22:256-63.

8. Swallow CJ, Strauss DC, Bonvalot S, et al. Management of primary retroperitoneal sarcoma (RPS) in the adult: an updated consensus approach from the Transatlantic Australasian RPS Working Group. Ann Surg Oncol. 2021. https://doi.org/10.1245/ s10434-021-09654-z.

9. Gronchi A, Strauss DC, Miceli R, et al. Variability in patterns of recurrence after resection of primary retroperitoneal sarcoma (RPS): a report on 1007 patients from the Multi-Institutional Collaborative RPS Working Group. Ann Surg. 2016;263:1002-9.

10. Dingley B, Fiore M, Gronchi A. Personalizing surgical margins in retroperitoneal sarcomas: an update. Expert Rev Anticancer Ther. 2019;19:613-31.

11. Bonvalot S, Gronchi A, Le Péchoux C, et al. Preoperative radiotherapy plus surgery versus surgery alone for patients with primary retroperitoneal sarcoma (EORTC-62092: STRASS): a multicentre, open-label, randomised, phase 3 trial. Lancet Oncol. 2020;21:1366-77.

Publisher's Note Springer Nature remains neutral with regard to jurisdictional claims in published maps and institutional affiliations. 